\title{
Drug-coated balloon superior to standard balloon angioplasty
}

Although endovascular therapy is the preferred treatment modality for patients with peripheral artery disease (PAD), the optimal revascularization strategy remains unclear. Two new randomized, controlled trials published in Circulation now report that drug-coated balloon (DCB) angioplasty is superior to uncoated balloon angioplasty for the treatment of patients with symptomatic PAD.

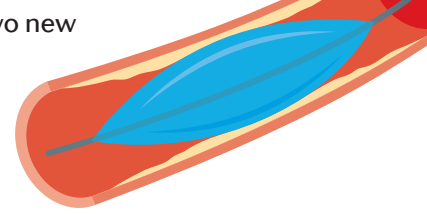

At present, balloon angioplasty with provisional stenting is the most widely adopted endovascular treatment strategy for PAD. Paclitaxel-eluting DCBs might be a viable alternative to drug-eluting stents, and have shown promising results in early studies. Investigators in the ILLUMENATE EU RCT trial compared the efficacy and safety of low-dose DCB angioplasty versus standard balloon angioplasty in patients with symptomatic PAD.

ILLUMENATE EU RCT was a multicentre, randomized, single-blind study involving patients with superficial femoral and/or proximal popliteal artery disease. Participants were randomly assigned (3:1) to angioplasty using a DCB (containing $2 \mu \mathrm{g} / \mathrm{mm}^{2}$ of paclitaxel) or a standard, uncoated balloon. The primary efficacy end point was primary vessel patency at 12 months, and the primary safety end point was a composite of freedom from device-related and procedure-related death at 30 days, and freedom from major-limb amputation and target-lesion revascularization at 12 months. Overall, 222 patients were assigned to the DCB group, and 72 to the uncoated balloon group. Lesion characteristics and procedural outcomes were not different between the two groups. DCBs were superior to uncoated balloons for both safety and efficacy outcomes.

According to the study investigators, ILLUMENATE EU RCT "is the first trial to demonstrate that angioplasty with a lose-dose DCB is able to achieve comparable clinical outcomes to that of a DCB with $75 \%$ more drug". Although the clinical implications of a higher dose of drug coating are not yet known, low-dose formulations "carry the potential to reduce distal drug embolization, which may translate into a safety advantage".

In a separate trial, the ISAR-STATH investigators compared the efficacy of a DCB plus stenting versus an uncoated balloon plus stenting versus directional atherectomy plus provisional stenting in patients with superficial femoral artery disease. The primary end point was angiography-assessed percentage diameter stenosis after 6 months. In total, 48 patients were assigned to the DCB group, 52 to the uncoated balloon group, and 55 to the directional atherectomy group. At 6 months, patients treated with DCB plus stenting had significantly lower percentage diameter stenosis $(34 \pm 31 \%)$ compared with either the uncoated balloon group ( $56 \pm 29 \%$; $P=0.009$ ), or the directional atherectomy group ( $55 \pm 29 \%$; $P=0.007$ ). Target-lesion revascularization at 24 months was also significantly lower in the DCB group than in the uncoated balloon group.

Together, the ILLUMENATE EU RCT and ISAR-STATH trials demonstrate superiority of DCB use compared with standard balloon angioplasty for the treatment of PAD. These findings support the broadening use of DCBs in patients with femoropopliteal disease.

Karina Huynh

ORIGINAL ARTICLES Schroeder, H. et al. Low-dose paclitaxel-coated versus uncoated percutaneous transluminal balloon angioplasty for femoropopliteal peripheral artery disease: 1 -year results of the ILLUMENATE European randomized clinical trial. Circulation http://dx.doi.org/10.1161/ CIRCULATIONAHA.116.026493 (2017) | Ott, I. et al. Randomized comparison of paclitaxel-eluting balloon angioplasty plus stenting versus standard balloon angioplasty plus stenting versus directional atherectomy for symptomatic femoral artery disease (ISAR-STATH). Circulation http://dx.doi.org/10.1161/

CIRCULATIONAHA.116.025329 (2017) 